\title{
Propane Vehicle and Infrastructure Codes and Standards Citations
}

This document lists codes and standards typically used for U.S. propane vehicle and infrastructure projects. To determine which codes and standards apply to a specific project, identify the codes and standards currently in effect within the jurisdiction where the project will be located. Some jurisdictions also have unique ordinances or regulations that could apply.

Learn about codes and standards basics at www.afdc.energy.gov/afdc/codes_standards_basics.html.

Find propane vehicle and infrastructure codes and standards in these categories:

- General Requirements

- Gas Equipment and Appliances

- Installation of LP Gas Systems

- LP-Gas Liquid Transfer

- Buildings or Structures Housing LP-Gas for Distribution

- Engine Fuel Systems

- Operations and Maintenance

- $\quad$ Piping and Tubing Sizing Tables

\section{General Requirements}

- NFPA 58, Liquefied Petroleum Gas Code, 4

\section{Acceptance of Equipment and Systems}

- NFPA 58, Liquefied Petroleum Gas Code, 4.1

\section{Odorization}

- NFPA 58, Liquefied Petroleum Gas Code, 4.2

\section{Notification of Installations}

- NFPA 58, Liquefied Petroleum Gas Code, 4.3

\section{Qualification of Personnel}

- NFPA 58, Liquefied Petroleum Gas Code, 4.4

\section{Ammonia Contamination}

- NFPA 58, Liquefied Petroleum Gas Code, 4.5

\section{Minimum Requirements}

- NFPA 58, Liquefied Petroleum Gas Code, 4.6

\section{Gas Equipment and Appliances}

- NFPA 58, Liquefied Petroleum Gas Code, 5 


\section{Containers}

- $\quad$ NFPA 58, Liquefied Petroleum Gas Code, 5.2

- ASME Boiler and Pressure Vessel Code, Section VIII, "Rules for the Construction of Unfired Pressure Vessels"

- API-ASME Code for Unfired Pressure Vessels for Petroleum Liquids and Gases,

- National Board Inspection Code

- CGA C-6, Standard for Visual Inspection of Steel Compressed Gas Cylinders

- ASCE 7, Minimum Design Loads for Buildings and Other Structures

\section{Container Appurtenances}

- $\quad$ NFPA 58, Liquefied Petroleum Gas Code, 5.7

- UL 132, Standard on Safety Relief Valves for Anhydrous Ammonia and LP-Gas

- UL 144, Standard for LP-Gas Regulators

- $\quad$ NFPA 54, National Fuel Gas Code (ANSI Z223.1), 5.9.2

- UL 651, Schedule 40 or 80 Rigid PVC Conduit

- CGA V-1, Standard Compressed Gas Cylinder Valve Outlet and Inlet Connections

- API 607, Fire Test for Soft-Seated Quarter Turn Ball Valves

\section{Piping (including Hose), Fittings and Valves}

- $\quad$ NFPA 58, Liquefied Petroleum Gas Code, 5.8

- NFPA 54, National Fuel Gas Code

- Referenced ASTM Piping and Tubing Standards

\section{Valves Other Than Container Valves}

- NFPA 58, Liquefied Petroleum Gas Code, 5.10

- Referenced ASTM Standards

\section{Hydrostatic Relief Valves}

- NFPA 58, Liquefied Petroleum Gas Code, 5.11

- Referenced ASTM Standards

\section{Equipment}

- NFPA 58, Liquefied Petroleum Gas Code, 5.15

- Referenced ASTM Standards

\section{Installation of LP Gas Systems}

- $\quad$ NFPA 58, Liquefied Petroleum Gas Code, 6 


\section{Scope}

- NFPA 58, Liquefied Petroleum Gas Code, 6.1

\section{Location of Containers}

- NFPA 58, Liquefied Petroleum Gas Code, 6.2

\section{Container Separation Distances}

- NFPA 58, Liquefied Petroleum Gas Code, 6.3

\section{Other Container Location Requirements}

- NFPA 58, Liquefied Petroleum Gas Code, 6.4

- NFPA 30, Flammable and Combustible Liquids Code

- NFPA 55, Compressed Gases and Cryogenic Fluids Code

- ANSI/CGA C-7, Guide to the Preparation of Precautionary Labeling and Marking of Compressed Gas Containers

\section{Location of Transfer Operations}

- NFPA 58, Liquefied Petroleum Gas Code, 6.5

\section{Installation of Containers}

- NFPA 58, Liquefied Petroleum Gas Code, 6.6

- NFPA 220, Standard on Types of Building Construction

\section{Installation of Containers Appurtenances}

- NFPA 58, Liquefied Petroleum Gas Code, 6.7

- ANSI Z21.80/CSA 6.22, Standard for Line Pressure Regulators.

- NFPA 54, National Fuel Gas Code

\section{Piping Systems}

- NFPA 58, Liquefied Petroleum Gas Code, 6.8

- ASME B 31.3, Process Piping

- ASME Boiler and Pressure Vessel Code, Section IX

- References ASTM Piping Standards

\section{Internal Valves}

- NFPA 58, Liquefied Petroleum Gas Code, 6.9

\section{Emergency Shutoff Valves}

- NFPA 58, Liquefied Petroleum Gas Code, 6.10

\section{Hydrostatic Relief Valve Installation}

- NFPA 58, Liquefied Petroleum Gas Code, 6.11

\section{Testing Piping Systems}

- NFPA 58, Liquefied Petroleum Gas Code, 6.12

- ASME Boiler and Pressure Vessel Code, Section IX

\section{Installation in Areas with Heavy Snowfall}

- NFPA 58, Liquefied Petroleum Gas Code, 6.13 
Corrosion Protection

- NFPA 58, Liquefied Petroleum Gas Code, 6.14

\section{Equipment Installation}

- NFPA 58, Liquefied Petroleum Gas Code, 6.15

\section{Bulk Plant and Industrial LP-Gas Systems}

- NFPA 58, Liquefied Petroleum Gas Code, 6.16

\section{LP Gas Systems in Buildings or on Roofs}

- NFPA 58, Liquefied Petroleum Gas Code, 6.17

- UL 147A, Standard for No refillable (Disposable) Type Fuel Gas Cylinder Assemblies

- UL 147B, Standard for No refillable (Disposal) Type Metal Container Assemblies for Butane

\section{Ignition Source Control}

- NFPA 58, Liquefied Petroleum Gas Code, 6.20

- NFPA 70, National Electrical Code

\section{Vehicle Fuel Dispenser and Dispensing Stations}

- NFPA 58, Liquefied Petroleum Gas Code, 6.22

- UL 567, Standard Pipe Connectors for Flammable and Combustible Liquids and LP-Gas

\section{Fire Protection}

- $\quad$ NFPA 58, Liquefied Petroleum Gas Code, 6.23

- NFPA 15, Standard for Water Spray Fixed Systems for Fire Protection

\section{Alternate Provisions for Installation of ASME Containers}

- $\quad$ NFPA 58, Liquefied Petroleum Gas Code, 6.24

\section{LP-Gas Liquid Transfer}

- $\quad$ NFPA 58, Liquefied Petroleum Gas Code, 7

\section{Scope}

- $\quad$ NFPA 58, Liquefied Petroleum Gas Code, 7.1

\section{Operational Safety}

- $\quad$ NFPA 58, Liquefied Petroleum Gas Code, 7.2

\section{Venting LP-Gas to the Atmosphere}

- NFPA 58, Liquefied Petroleum Gas Code, 7.3

\section{Quantity of LP-Gas in Containers}

- $\quad$ NFPA 58, Liquefied Petroleum Gas Code, 7.4 


\section{Buildings or Structures Housing LP-Gas for Distribution}

- NFPA 58, Liquefied Petroleum Gas Code, 10

\section{Scope}

- $\quad$ NFPA 58, Liquefied Petroleum Gas Code, 10.1

\section{Separate Structures or Buildings}

- $\quad$ NFPA 58, Liquefied Petroleum Gas Code, 10.2

- NFPA 70, National Electrical Code

Attached Structures or Rooms Within Structures

- NFPA 58, Liquefied Petroleum Gas Code, 10.3

\section{Engine Fuel Systems}

- NFPA 58, Liquefied Petroleum Gas Code, 11

\section{Scope}

- NFPA 58, Liquefied Petroleum Gas Code, 11.1

\section{Training}

- NFPA 58, Liquefied Petroleum Gas Code, 11.2

\section{Containers}

- NFPA 58, Liquefied Petroleum Gas Code, 11.3

- U.S. Department of Transportation (DOT)

- Rules for Construction of Unfired Pressure Vessels," Section VIII, Division I of the ASME Boiler and Pressure Vessel Code

\section{Container Appurtenances}

- NFPA 58, Liquefied Petroleum Gas Code, 11.4

- UL 132, Standard on Safety Relief Valves for Anhydrous Ammonia and LP-Gas

\section{Carburetion Equipment}

- $\quad$ NFPA 58, Liquefied Petroleum Gas Code, 11.5

\section{Piping, Hose, and Fittings}

- $\quad$ NFPA 58, Liquefied Petroleum Gas Code, 11.6

- Referenced ASTM Piping and Tubing Standards

Installation of Containers and Container Appurtenances

- $\quad$ NFPA 58, Liquefied Petroleum Gas Code, 11.7

Installation in the Interior of Vehicles

- NFPA 58, Liquefied Petroleum Gas Code, 11.8 
Pipe and Hose Installation

- NFPA 58, Liquefied Petroleum Gas Code, 11.9

\section{Equipment Installation}

- NFPA 58, Liquefied Petroleum Gas Code, 11.10

\section{Marking}

- NFPA 58, Liquefied Petroleum Gas Code, 11.11

Industrial (and Forklift) Trucks Powered by LP-Gas

- NFPA 58, Liquefied Petroleum Gas Code, 11.12

General Provisions for Vehicles Having Engines Mounted on Them

- NFPA 58, Liquefied Petroleum Gas Code, 11.13

\section{Engine Installation Other Than on Vehicle}

- NFPA 58, Liquefied Petroleum Gas Code, 11.14

\section{Garaging of Vehicles}

- NFPA 58, Liquefied Petroleum Gas Code, 11.15

\section{Operations and Maintenance}

- NFPA 58, Liquefied Petroleum Gas Code, 14

\section{Scope}

- $\quad$ NFPA 58, Liquefied Petroleum Gas Code, 14.1

Operating Requirements

- NFPA 58, Liquefied Petroleum Gas Code, 14.2

\section{Maintenance}

- NFPA 58, Liquefied Petroleum Gas Code, 14.3

- NFPA 25, Standard for the Inspection, Testing, and Maintenance of Water-Based Fire Protection Systems

- NFPA 10, Standard for Portable Fire Extinguishers

\section{Piping and Tubing Sizing Tables}

- NFPA 58, Liquefied Petroleum Gas Code, 15 